\title{
Validez y confiabilidad de la Escala de Felicidad de Lima en adultos mayores venezolanos
}

\author{
Validity and reliability of the Scale of Happiness \\ from Lima in Venezuelan elderly people
}

Recibido: mayo 5 de 2010 | Revisado: noviembre 3 de 2010 | Aceptado: enero 3 de 2011

\author{
MARISELA ViRginia ÁrRaga BarRios ** \\ MARHILDE SÁNCHEZ VILLARROEL ${ }^{* * *}$ \\ Universidad de Zulia, Maracaibo, Venezuela
}

\section{RES U MEN}

Con el propósito de determinar las propiedades psicométricas: validez y confiabilidad, de la Escala de Felicidad de Lima, para ser aplicada en adultos mayores venezolanos, se realizó un estudio descriptivo, experimental, de campo y transeccional, con 103 adultos mayores venezolanos entre 60 y 85 años. Los procedimientos estadísticos utilizados fueron el análisis factorial para evaluar su validez, el coeficiente alfa de Cronbach y el coeficiente partición por mitades, para la confiabilidad. Como resultado se obtuvo una "Versión adaptada de la Escala de Felicidad de Lima para adultos mayores" constituida por 14 ítems agrupados en cuatro dimensiones que miden el constructo felicidad. Los valores psicométricos obtenidos la acreditan como un instrumento válido y confiable para ser aplicado a adultos mayores. Palabras clave autores

Validez, confiabilidad, felicidad, adultos mayores.

Palabras clave descriptores

Para citar este artículo. Árraga, M. V. \& Sánchez, M. (2012). Validez y confiabilidad de la Escala de Felicidad de Lima en adultos mayores venezolanos. Universitas Psychologica, 11(2), 381-393.

Este estudio constituye una primera etapa de la investigación titulada "Bienestar subjetivo del adulto mayor venezolano", para la cual se requirió efectuar el estudio técnico del instrumento de recolección de datos.

** Profesora Titular e investigadora del Departamento de Psicología, Escuela de Educación, Facultad de Humanidades y Educación de la Universidad del Zulia (LUZ). E-mail: mariarraga@hotmail.com ResearcherID: Árraga, M. 3315-2012.

**** Profesora Titular e investigadora del Departamento de Psicología, Escuela de Educación, Facultad de Humanidades y Educación de la Universidad del Zulia (LUZ).E-mail: marsanchezg@cantv.net

\section{A B S T R A C T}

With the purpose to determine the psychometric properties: validity and reliability, of the Scale of Happiness from Lima, applied to Venezuelan elderly people, it was made a descriptive study, experimental, of field and transeccional, with 103 elderly people between 60 and 85 years old. The statistics procedures were factorial analysis to evaluate validity and for the reliability, the coefficient Cronbach's Alpha and the coefficient Split-half. As result was obtained an instrument called "Revised version of the Scale of Happiness from Lima for elderly people". It was composed of 14 items grouped into four dimensions through which the Happiness construct is measured. The psychometric values obtained with the Scale accredit it as a valid and reliable instrument.

Key words authors

Validity, reliability, happiness, elderly people.

Key words plus

Psychological tests, psychometry, quantitative research. 


\section{Introducción}

La felicidad es un concepto que ha sido utilizado profusamente en la literatura y en diversas áreas del quehacer científico. La Psicología no se ha escapado a esto y en las últimas décadas la inclusión de la felicidad en las investigaciones psicológicas, ha proliferado, más aún con el surgimiento de la flamante Psicología Positiva.

No obstante, el uso de este término ha sido un tanto ambiguo y difuso en la bibliografía científica, debido a su inaprensibilidad y a las connotaciones filosóficas de las que ha sido objeto. En 1973, el término felicidad fue introducido en el Psychological Abstract International, pero debido precisamente a su carácter difuso y a las connotaciones filosóficas que se le han asignado, desde entonces, se ha tratado de definir de una manera más operativa buscando un concepto válido científicamente, lo que ha dado paso en el vocabulario psicológico al concepto de "bien-estar" (well-being) o de "satisfacción con la vida” (Fierro, 2000 citado por García, 2002).

$\mathrm{Al}$ respecto, se ha encontrado que para 1967, Warner Wilson hizo una amplia revisión del bienestar subjetivo, concluyendo que la persona feliz es joven, saludable, bien educada, bien pagada, extrovertida, optimista, libre, religiosa, casada, con alta autoestima, moral de trabajo, aspiraciones modestas, de cualquier sexo y de un amplio rango de inteligencia (Diener, Suh, Lucas \& Smith, 1999). Con estas conclusiones, el constructo felicidad comienza a convertirse en sinónimo de bienestar subjetivo.

Para 1974, en los Estados Unidos de América, se crea el Social Indicators Research, revista especializada en el estudio de la calidad de vida y del bienestar personal, con una fuerte tradición economicista que inicialmente tuvo una orientación fundamentalmente objetiva, con énfasis en el aspecto socioeconómico y de bienestar social. Paralelamente, durante las pasadas décadas, un gran número de investigadores se dedicaron a analizar correlatos demográficos y variables vinculadas con el bienestar humano.

Esto ha dado lugar a estudios en los que se examinan las diferencias personales respecto a la felicidad, atendiendo otros factores tales como la actuación y percepción que tiene la persona de su propia vida, su estado de ánimo, el nivel afectivo y la satisfacción vital, entre otros (García, 2002). A partir de entonces, las investigaciones sobre bienestar subjetivo y/o felicidad han ido avanzando y evolucionando, así como los métodos que se han utilizado para llevarlas a cabo.

El aporte de la Psicología Positiva ha sido significativo, por cuanto su inclinación a investigar acerca de las fortalezas y virtudes humanas y los efectos que estas tienen en las vidas de las personas y en las sociedades en las que viven, ha despertado un interés particular por indagar rasgos de la persona relacionados con la capacidad para el amor y la vocación, el valor, las habilidades interpersonales, la sensibilidad estética, la perseverancia, el perdón, la originalidad, la espiritualidad, el talento, la sabiduría, la satisfacción con la vida, entre otros, incluyendo la felicidad y el bienestar subjetivo (Cuadra \& Florenzano, 2003).

Con la finalidad de indagar sobre algunos de estos aspectos, se han elaborado diversos instrumentos para evaluar y medir felicidad o bienestar subjetivo. En lo que respecta a definir instrumento de medición, Hernández, Fernández y Baptista (2005) lo refieren como el recurso que utiliza el investigador para registrar información o datos sobre las variables de interés. Así mismo, Rodríguez y Pineda (2003 citados por Pelekais, Finol, Newman \& Parada, 2005) lo describen como el recurso del que se vale el investigador para acercarse a la realidad y extraer información sobre su objeto de estudio. Estos autores también señalan su utilidad en el registro de datos objetivos o subjetivos, primarios o secundarios, que se encuentran en el contexto estudiado.

De acuerdo a Alarcón (2006), los instrumentos más conocidos y acreditados que miden estos constructos son: el Oxford Happiness Inventory (OHI) construido por Martin y Crossland, el Oxford Happiness Questionaire de Hills y Argyle, la Satisfaction with Life Scale desarrollado por Diener, Emmons, Larsen y Griffin, que tiene una versión posterior modificada por el propio Diener y Pavot; también se conoce la escala Depresion-Happiness Scale (DHS), la Memorial University of Newfound- 
land Scale of Happiness y el Chinese Happiness Inventory, basado en el Oxford Happiness Inventory.

En cuanto a las versiones en español, continúa el autor, se conocen muy pocos instrumentos de medición, uno de ellos es la Escala de Bienestar Psicológico de Sánchez-Canovas, y la también reconocida Escala de Bienestar Subjetivo de Albuquerque y Tróccoli, construída en portugués.

Pelekais et al. (2005), por su parte, enuncian la importancia de cumplir con el proceso técnico de validez y confiabilidad en toda prueba o instrumento de carácter científico. Referente a la validez, Hernández et al. (2005, p. 277) la definen como "el grado en el que un instrumento realmente mide la variable que pretende medir". De dicho concepto pueden presentarse distintos tipos de evidencia: la vinculada con el contenido, la relacionada con el criterio y la referida al constructo. Esta última se considera la más difícil y atañe a las pruebas psicológicas que afirman medir atributos psicológicos complejos, multifacéticos y vinculados con la teoría, por lo que establecerla es un proceso largo y complejo. Su interés suele centrarse más en las propiedades que están siendo medidas que en la prueba empleada para llevar a cabo la medición (Kerlinger, 2002).

Señala Gregory (2001) que muchos teóricos en psicometría, tales como Cronbach en 1988, Guion en 1980 y Messick en 1995, consideran a la validez de constructo el concepto unificador para todos los tipos de evidencia de validez por lo que, según esta perspectiva, los estudios individuales de validez de contenido, concurrente y predictiva, se consideran solo como evidencia de apoyo, en la búsqueda acumulativa de validación del constructo.

El análisis factorial es una técnica estadística especializada, de particular utilidad para la investigación de la validez de constructo. El análisis factorial confirmatorio tiene como propósito, según Gregory (2001), ratificar que las calificaciones de una prueba y las variables se articulan en cierto patrón predicho por una teoría, con el propósito de generar una descripción económica de conjuntos amplios y complejos de datos. Presenta como punto inicial, la matriz de correlación y una tabla completa de intercorrelaciones entre todas las variables.
En la presente investigación, al indagar sobre la validez de constructo, se manifiesta el sesgo en ese tipo de validez, refiriéndose a cuando se demuestra que una prueba mide diferentes rasgos hipotéticos (constructos psicológicos) en un grupo en relación con otro, o mide el mismo rasgo, pero con diferentes grados de precisión (Reynolds, 1982 citado por Gregory, 2001).

Esto ocurrió cuando en el presente estudio se administró la Escala de Felicidad de Lima de Reynaldo Alarcón (2006) a una población de adultos mayores (personas de 60 años o más) y, al efectuar el análisis factorial del instrumento, los resultados fueron diferentes a los obtenidos por el propio Alarcón, quien lo aplicó a una población de adultos jóvenes. Al vincular este resultado a lo expuesto por Gregory (2001), se observa un sesgo en la validez de constructo cuando al comparar dos subpoblaciones pertinentes, estas no revelaron un alto grado de semejanza en la estructura factorial de la prueba.

Es importante destacar como un criterio esencial de la imparcialidad, que la estructura factorial de las calificaciones deba ser invariable entre subpoblaciones apropiadas; en particular, en el caso de una prueba imparcial, el número de factores que surgen y las cargas factoriales de los reactivos o subescalas serán sumamente similares en las subpoblaciones relacionadas. Por consiguiente, al no ser estas cargas factoriales de los reactivos similares, resultó una adaptación de la prueba original, para ser administrada a los adultos mayores.

Aunado a esto, es relevante enfatizar cómo ningún instrumento de medición puede tener valor a menos que mida algo en forma consistente o confiable. Por esto, otro de los primeros aspectos que se deben determinar es si las pruebas son lo suficientemente confiables o no, para medir aquello para lo cual se crearon.

Chávez (2001, p. 75) define la confiabilidad como el "grado de congruencia con que se realiza la medición de una variable". Si en ausencia de cualquier cambio permanente en una persona (producto del crecimiento, aprendizaje, enfermedad o accidente), las calificaciones de las pruebas varían en gran medida de tiempo en tiempo o en diferentes situaciones, es probable que la prueba no 
sea confiable y no pueda utilizarse para explicar o realizar predicciones sobre el comportamiento de las personas (Aiken, 2003).

Santrock (2004) señala que una prueba estable y consistente no debe fluctuar de manera significativa debido a factores aleatorios como: cuánto durmió la noche anterior la persona que respondió la prueba, quién es el examinador o cuál es la temperatura del lugar donde se aplica la prueba.

Cohen y Swerdlik (2006) y Gregory (2001, p. 122) definen la confiabilidad como "el atributo de consistencia en la medición”, considerándola siempre como un intento por estimar la probable precisión o repetibilidad de las puntuaciones de prueba. La confiabilidad es un elemento necesario, pero no suficiente, de una buena prueba; de manera que, además de ser confiables, las pruebas deben ser precisas de una manera razonable, es decir, deben ser válidas (Cohen \& Swerdlik, 2006).

Según Papalia y Olds (2004), se han diseñado varios métodos estadísticos para estimar el grado de confiabilidad de las mediciones, fiabilidad testretest, en la cual se aplica la misma prueba al mismo grupo o persona, más de una vez. También exponen el método de formas alternativas o paralelas del test, con elevadas similitudes en formato, contenido y nivel de dificultad y el método de las dos mitades. Dicho enfoque de una sola aplicación de las pruebas, descrito por Gregory (2001) para estimar la correlación de los pares de puntuaciones obtenidas de mitades equivalentes de una prueba administrada en una única oportunidad a una muestra representativa de examinados, consiste específicamente en calcular una $r$ de Pearson entre las puntuaciones de las dos mitades equivalentes de la prueba, e implica un paso adicional: ajustar la confiabilidad utilizando la fórmula de Spearman-Brown.

Así mismo, el coeficiente alfa consiste en la media de todos los posibles coeficientes por mitades, corregidos según la fórmula de Spearman-Brown, la cual es un índice de consistencia interna de los reactivos, es decir, la tendencia a correlacionarse en sentido positivo entre sí. Por lo general, el test se divide en dos partes, correspondientes a las preguntas pares e impares, dicho método genera una medida de consistencia interna del instrumento.
En esta investigación se utiliza este último método, para comprobar la confiabilidad de la Escala de Felicidad de Lima, elaborada por Alarcón para la población de América Latina, al ser administrada a adultos mayores venezolanos.

Uno de los elementos que motivó a la realización del trabajo es la escasez de instrumentos elaborados para medir felicidad en y para los países latinoamericanos, así como la evidencia encontrada en diferentes investigaciones acerca de la influencia de los factores culturales sobre el significado subjetivo que las personas le atribuyen a las cosas, y sobre la conceptualización teórica de la conducta. Esto ha generado la necesidad de construir instrumentos que se adecuen a las características culturales de cada país.

Como respuesta a esta inquietud, el profesor Reynaldo Alarcón de la Universidad Ricardo Palma en Lima, Perú, construyó la llamada Escala de Felicidad de Lima. Para ello, definió operacionalmente el constructo felicidad mediante cuatro factores y sus respectivos indicadores, a través de una serie de ítems. Luego de redactados estos ítems, comprobó su validez de contenido y los organizó en una escala experimental tipo Likert de cinco alternativas, que fue suministrada a 709 estudiantes universitarios, de la ciudad de Lima, hembras y varones con una edad media de 23.1 años (Alarcón, 2006).

El término felicidad del que se partió para la construcción de esta escala y que se utiliza en este estudio, se define como un estado afectivo de satisfacción plena que experimenta subjetivamente una persona y que se considera sinónimo de bienestar subjetivo. Esta definición está inspirada en las ideas filosóficas de felicidad del mundo griego, en particular Aristóteles y Epicuro, y en los hallazgos recientes de la investigación empírica de la felicidad o subjective well-being, tal como lo expresa Alarcón (2006).

Como constructo, la felicidad está compuesta por cuatro factores, los cuales conforman la Escala de Felicidad de Lima elaborada por Alarcón, son ellos:

1. Sentido positivo de la vida: implica tener sentimientos positivos hacia sí mismo y hacia la 
vida, estando exentos de estados depresivos profundos. O, como lo explican Havighurst, Neugarten y Tobin (1999), se refiere a una valoración del afecto positivo frente al nivel de actividad en los roles de la vida cotidiana.

2. Satisfacción con la vida: refiere estados subjetivos positivos de satisfacción de las personas. Para Yuste, Rubio y Aleixandre (2004) no depende solamente de condiciones presentes, sino también de perspectivas futuras.

3. Realización personal: supone la orientación de la persona hacia metas que considera valiosas para su vida.

4. Alegría de vivir: referido a ver las experiencias de la vida en forma positiva y sentirse generalmente bien.

Una vez elaborado el instrumento Escala de Felicidad de Lima por Alarcón y suministrado a la muestra seleccionada, se procedió a realizar el análisis psicométrico, la validez de constructo y el análisis factorial del mismo, dando como resultado definitivo una escala tipo Likert compuesta por 27 ítems con cinco alternativas de respuestas: totalmente de acuerdo, acuerdo, ni acuerdo ni desacuerdo, desacuerdo y totalmente en desacuerdo, donde al extremo positivo se le otorgan cinco puntos y al negativo un punto.

Los valores psicométricos obtenidos con la aplicación de esa escala, la acreditan como un instrumento válido y confiable; así mismo, la muestra poblacional con la que se trabajó en su construcción (adultos jóvenes residentes en Lima) permitió adaptarlo a las características de la población latina, por lo que se constituye en una herramienta que puede realizar importantes contribuciones al desarrollo de investigaciones sobre la felicidad en América Latina.

Con base en la afirmación de adecuar los instrumentos de medición psicológica a las características de la población con la que se pretende trabajar, y en el empeño personal de trabajar con adultos mayores, se diseñó este estudio con el propósito de determinar las propiedades psicométricas: validez y confiabilidad, de la Escala de Felicidad de Lima, al ser aplicada a adultos mayores venezolanos. Es- ta inquietud responde a que con anterioridad se realizaron algunos estudios para definir y medir el bienestar subjetivo de las personas mayores, generalmente con el objetivo de utilizar esta medida como una definición operativa de lo que es envejecer con éxito.

Varios términos han sido utilizados para tratar la situación de estudio (términos tales como ajuste, competencia, moral o felicidad), así como diferentes criterios y técnicas de medición; sin embargo, uno de los elementos importantes que no ha sido considerado es que, tal vez, no es adecuado medir el bienestar subjetivo en la tercera edad utilizando los mismos estándares aplicables a la edad mediana o joven, ya que estos últimos se basan en la actividad y el compromiso social (Havighurst et al., 1999). De allí surge la inquietud de medir o evaluar la felicidad o bienestar subjetivo en adultos mayores, con un instrumento acorde con las características de esta población.

\section{Método}

Este estudio constituye una primera etapa de la investigación titulada "Bienestar subjetivo del adulto mayor venezolano", para la cual se requirió efectuar el estudio técnico del instrumento de recolección de datos: validez (análisis factorial) y confiabilidad, mediante la presente investigación de tipo descriptivo, con un diseño no experimental, de campo, transeccional. La investigación se llevó a cabo en Venezuela, con la participación de 103 adultos mayores o ancianos, cuyo rango de edad fue entre 60 y 85 años, residentes en instituciones geriátricasgerontológicas y que viven en sus hogares o con sus familiares. No se discriminó en los participantes género ni nivel socioeconómico.

El instrumento original con el que se trabajó fue la Escala de Felicidad de Lima (Alarcón, 2006) integrada por 27 ítems y cinco opciones de respuestas predeterminadas: Totalmente de Acuerdo (TA), Acuerdo (A), Ni acuerdo ni en desacuerdo (i), en Desacuerdo (D) y Totalmente en Desacuerdo (TD), aplicada de manera individual a los adultos mayores en sus lugares de residencia, por parte de las autoras de la investigación. 
TABLA 1

Varianza total explicada

\begin{tabular}{|c|c|c|c|c|c|c|c|c|c|}
\hline \multirow[t]{2}{*}{ Componente } & \multicolumn{3}{|c|}{$\begin{array}{l}\text { Autovalores } \\
\text { iniciales }\end{array}$} & \multicolumn{3}{|c|}{$\begin{array}{l}\text { Sumas de las saturaciones } \\
\text { al cuadrado de la extracción }\end{array}$} & \multicolumn{3}{|c|}{$\begin{array}{l}\text { Suma de saturaciones } \\
\text { al cuadrado de rotación }\end{array}$} \\
\hline & Total & $\begin{array}{c}\% \\
\text { varianza }\end{array}$ & $\begin{array}{c}\% \\
\text { acumul. }\end{array}$ & Total & $\begin{array}{c}\% \\
\text { varianza }\end{array}$ & $\begin{array}{c}\% \\
\text { acumul. }\end{array}$ & Total & $\begin{array}{c}\% \\
\text { varianza }\end{array}$ & $\begin{array}{c}\% \\
\text { acumul. }\end{array}$ \\
\hline 1 & 4.914 & 35.1 & 35.1 & 4.914 & 35.1 & 35.1 & 3.548 & 25.344 & 25.344 \\
\hline 2 & 1.39 & 9.932 & 45.032 & 1.39 & 9.932 & 45.032 & 1.813 & 12.95 & 38.295 \\
\hline 3 & 1.255 & 8.967 & 53.999 & 1.255 & 8.967 & 53.999 & 1.676 & 11.972 & 50.266 \\
\hline 4 & 1.034 & 7.388 & 61.387 & 1.034 & 7.388 & 61.387 & 1.557 & 11.12 & 61.387 \\
\hline 5 & 0.907 & 6.481 & 67.867 & & & & & & \\
\hline 6 & 0.749 & 5.353 & 73.22 & & & & & & \\
\hline 7 & 0.671 & 4.795 & 78.016 & & & & & & \\
\hline 8 & 0.598 & 4.269 & 82.284 & & & & & & \\
\hline 9 & 0.559 & 3.99 & 86.274 & & & & & & \\
\hline 10 & 0.528 & 3.772 & 90.046 & & & & & & \\
\hline 11 & 0.432 & 3.087 & 93.133 & & & & & & \\
\hline 12 & 0.363 & 2.594 & 95.727 & & & & & & \\
\hline 13 & 0.319 & 2.276 & 98.003 & & & & & & \\
\hline 14 & 0.28 & 1.997 & 100 & & & & & & \\
\hline
\end{tabular}

Fuente: elaboración propia.

Es importante destacar que la mayoría de los adultos mayores respondieron la escala, sin embargo, a algunos fue necesario leerles los enunciados y/o escribir las respuestas, producto de limitaciones visuales o motoras y analfabetismo. Posteriormente, se tabularon los datos, asignándole a los ítems positivos 5 puntos al nivel mayor de felicidad y 1 punto al nivel más bajo, con los ítems negativos se hizo en sentido inverso.

Se efectuaron los procedimientos estadísticos requeridos: la validez de la escala fue evaluada por medio del análisis factorial de acuerdo al procedimiento señalado por Alarcón, utilizándose el método de componentes principales para marcar el número de factores de la solución, y la rotación Varimax para controlar y extraer la solución idónea al mismo. La confiabilidad se obtuvo con el coeficiente alfa de Cronbach y el coeficiente partición por mitades, con el procedimiento de SpearmanBrown (longitudes iguales) y el de Guttman.

\section{Resultados}

\section{Validez Factorial de la Escala}

El análisis de componentes principales con cuatro componentes señala valores Eigen que varían de 1.03 a 4.91. El primer componente explica el $25.34 \%$ de la varianza, en tanto la suma de los cuatro componentes es el $61.38 \%$, tal como se aprecia en la Tabla 1 de la varianza total explicada.

La rotación ortogonal Varimax agrupa los 27 reactivos en los cuatro componentes principales, observándose saturaciones altas de los factores en solo 14 de los ítems (Tabla 2).

Los 14 reactivos con mayores saturaciones que fueron extraídos son los ítems: 1, 2, 3, 5, 6, 8, 9, $10,12,16,17,18,21$ y 26 , tal como se muestra en la Tabla 3. En el tratamiento estadístico, se utilizó como método de extracción el Análisis de compo- 
TABLA 2

Matriz de componentes rotados (a)

\begin{tabular}{|c|c|c|c|c|}
\hline Componente & 1 & 2 & 3 & 4 \\
\hline Ítem 13 & 0.783 & 0.168 & 0.18 & 0.23 \\
\hline Ítem 11 & 0.707 & 0.131 & 0.206 & 0.145 \\
\hline Ítem 25 & 0.668 & 0.197 & 0.221 & \\
\hline Ítem 12 & 0.644 & 0.16 & & 0.459 \\
\hline Ítem 6 & 0.548 & 0.136 & 0.295 & 0.421 \\
\hline Ítem 10 & 0.546 & 0.219 & 0.239 & 0.448 \\
\hline Ítem 15 & 0.382 & 0.337 & 0.367 & -0.143 \\
\hline Ítem 23 & 0.248 & 0.801 & 0.19 & \\
\hline Ítem 22 & & 0.776 & 0.108 & 0.292 \\
\hline Ítem 17 & 0.278 & 0.656 & & 0.122 \\
\hline Ítem 20 & 0.214 & 0.644 & & 0.347 \\
\hline Ítem 18 & 0.332 & 0.605 & & \\
\hline Ítem 2 & -0.131 & 0.602 & 0.238 & 0.167 \\
\hline Ítem 26 & & 0.57 & 0.299 & 0.133 \\
\hline Ítem 7 & 0.309 & 0.479 & 0.302 & 0.122 \\
\hline Ítem 1 & & 0.133 & 0.784 & -0.119 \\
\hline Ítem 4 & 0.194 & & 0.696 & 0.414 \\
\hline Ítem 24 & 0.477 & 0.11 & 0.53 & 0.221 \\
\hline Ítem 9 & 0.437 & & 0.471 & \\
\hline Ítem 5 & 0.428 & 0.104 & 0.468 & \\
\hline Ítem 16 & 0.244 & 0.273 & 0.438 & 0.429 \\
\hline Ítem 27 & 0.179 & & 0.421 & 0.13 \\
\hline Ítem 14 & & 0.219 & & 0.73 \\
\hline Ítem 19 & 0.197 & 0.549 & & 0.633 \\
\hline Ítem 8 & 0.393 & & 0.24 & 0.545 \\
\hline Ítem 3 & 0.18 & & 0.484 & 0.508 \\
\hline Ítem 21 & 0.358 & 0.219 & 0.348 & 0.454 \\
\hline
\end{tabular}

Fuente: elaboración propia.

nentes principales, y como método de rotación, la Normalización Varimax con Kaiser.

De acuerdo a estos resultados, se encuentra una alta saturación factorial en 14 ítems, los cuales se reagrupan en las cuatro dimensiones referidas por Alarcón, así: Factor 1: Satisfacción con la vida, Factor 2: Realización personal, Factor 3: Sentido positivo de la vida y Factor 4: Alegría de vivir, garantizando la convergencia entre las medidas refe- ridas al constructo felicidad con menos cantidad de reactivos que la versión original de la escala, al ser aplicada a adultos mayores. El Factor 1 concentra siete ítems; el Factor 2 reúne tres; los Factores 3 y 4, dos reactivos cada uno (Tabla 4).

Este resultado permitió adaptar la Escala de Felicidad de Lima, construida por Alarcón, a la población de adultos mayores venezolanos, resultando un instrumento compuesto por 14 ítems que 
se califica atribuyéndole 5 puntos al nivel más alto de felicidad y 1 punto al nivel más bajo. Los niveles intermedios reciben los demás puntajes.

TABLA 3

Matriz de componentes rotados (a)

\begin{tabular}{lcccc}
\hline \multicolumn{5}{c}{ Componente } \\
\hline & 1 & 2 & 3 & 4 \\
\hline Ítem 10 & 0.809 & & & 0.219 \\
Ítem 6 & 0.723 & 0.184 & 0.197 & 0.122 \\
Ítem 8 & 0.704 & 0.161 & & -0.144 \\
Ítem 12 & 0.694 & & 0.394 & -0.110 \\
Ítem 21 & 0.643 & 0.195 & & 0.210 \\
Ítem 16 & 0.606 & 0.297 & & 0.364 \\
Ítem 3 & 0.562 & 0.247 & & 0.309 \\
Ítem 5 & 0.230 & 0.749 & 0.228 & \\
Ítem 1 & & 0.746 & & 0.358 \\
Ítem 9 & 0.382 & 0.654 & 0.116 & \\
Ítem 18 & 0.152 & 0.108 & 0.797 & 0.147 \\
Ítem 17 & 0.169 & & 0.796 & 0.216 \\
Ítem 26 & 0.207 & & & 0.735 \\
Ítem 2 & & & 0.318 & 0.664 \\
\hline
\end{tabular}

Fuente: elaboración propia.

Los ítems negativos de la escala son: $2,17,18$ y 26, mientras que los positivos son: $1,3,5,6,8,9,10$, 12,16 y 21. Altas puntuaciones reflejan reacciones positivas a la felicidad y bajas puntuaciones indican baja felicidad. Para responder esta escala, la persona debe elegir entre cinco alternativas de respuestas: $\mathrm{TA}=$ Totalmente de acuerdo, $\mathrm{A}=\mathrm{Acuerdo}, i=\mathrm{Ni}$ de acuerdo ni en desacuerdo, $\mathrm{D}=$ Desacuerdo y $\mathrm{TD}=$ Totalmente en desacuerdo (Apéndice).

\section{Coeficientes de confiabilidad}

El resultado obtenido con el coeficiente de alfa de Cronbach indica que los valores para cada uno de los ítems de la escala quedaron establecidos en un rango de 0.82 y de 0.84 , tal como lo explica la Tabla 5 .

La Tabla 6 muestra el resultado del coeficiente de partición por mitades de Spearman-Brown (lon- gitudes iguales) $r=0.89$ y el de Guttman $r=0.89$; ambos valores son altos y respaldan la alta confiabilidad de la escala analizada.

\section{Discusión}

En nuestro propósito de continuar investigando sobre los adultos mayores, tal como se ha reflejado en la publicación de libros y artículos científicos como Orientación gerontológica: desarrollo humano y calidad de vida en la vejez, Identidad de ancianos marabinos, Recreación y calidad de vida en adultos mayores que viven en instituciones geriátricas y en sus hogares: un estudio comparativo, entre otros, quisimos indagar sobre la felicidad de este grupo etario, tomando en consideración la Escala de Felicidad de Lima diseñada por Alarcón (2006).

Para ello, tuvimos la inquietud de conocer las propiedades psicométricas de dicha escala a fin de determinar o verificar si el instrumento podía ser utilizado para medir dicha variable (felicidad) en los adultos mayores, ya que cuando se construyó, la población estuvo constituida por jóvenes latinoamericanos.

Por tal razón, al administrar el instrumento original a un grupo de adultos mayores venezolanos, se determinó la validez factorial mediante el método de componentes principales, para marcar el número de factores de la solución y la rotación Varimax con el fin de controlar y extraer la solución idónea al mismo.

$\mathrm{Al}$ analizar los resultados se obtuvo una saturación alta solo en 14 de los 27 reactivos que componen la escala original, que según su comportamiento quedaron agrupados en los cuatro factores denominados dimensiones, sugeridos por Alarcón para medir felicidad. Las derivaciones del análisis factorial permitieron reagrupar los reactivos en las cuatro dimensiones que constituyen el constructo felicidad, de la siguiente manera:

Factor 1: Satisfacción con la vida. Los ítems reagrupados en esta dimensión son: 10, 6, 8, 12, 21, 16 y 3 , relativos a la expresión de satisfacción por los logros alcanzados y la creencia de que se está donde se tiene que estar. 
TABLA 4

Reagrupación por factores de ítems con altas saturaciones

\begin{tabular}{lcc}
\hline & \multicolumn{2}{c}{ Componente } \\
\hline & 1 & 2 \\
\hline 10. Me siento satisfecho porque estoy donde tengo que estar. & 0.809 \\
6. Me siento satisfecho con lo que soy. & 0.723 & \\
8. Hasta ahora, he conseguido las cosas que para mí son importantes. & 0.704 \\
12. Es maravilloso vivir. & 0.694 \\
21. Estoy satisfecho con lo que hasta ahora he alcanzado. & 0.643 \\
16. He experimentado la alegría de vivir. & 0.606 & 0.562 \\
3. Las condiciones de mi vida son excelentes. & 0.749 \\
5. La vida ha sido buena conmigo. & 0.746 \\
1. En la mayoría de las cosas, mi vida está cerca de mi ideal. & 0.654 \\
9. Si volviese a nacer no cambiaría nada en mi vida. & & 0.797 \\
18. Tengo problemas tan hondos que me quitan la tranquilidad. & & 0.796 \\
17. La vida ha sido injusta conmigo. & & \\
26. Todavía no he encontrado sentido a mi existencia. & & \\
2. Siento que mi vida esta vacía. & & \\
\hline
\end{tabular}

Fuente: elaboración propia.

Factor 2: Realización personal. Los ítems reagrupados para esta dimensión son: 5, 1 y 9, los cuales señalan lo que se puede llamar felicidad plena y no estados temporales de la misma.

Factor 3: Sentido positivo de la vida. Los ítems reagrupados en esta dimensión son: 18 y 17, y tal como están formulados indican depresión, fracaso, pesimismo y vacío existencial. Las respuestas de rechazo a lo que afirman las oraciones indican grados de felicidad.

Factor 4: Alegría de vivir. Los ítems reagrupados en esta dimensión son: 26 y 2. Las respuestas de repudio señalan lo maravilloso que es vivir, expresando experiencias positivas de la vida y sentirse generalmente bien.

Al aplicar la validez factorial a la escala original de Alarcón, administrada a un grupo de adultos mayores venezolanos para corroborar la validez de constructo, el número de factores que surgieron y las cargas factoriales de los reactivos fueron diferentes a los alcanzados con la población de adultos jóvenes que administró el autor, por lo que hubo variabilidad entre las subpoblaciones.

Debido a que estas cargas factoriales de los reactivos no son similares, resultó una Escala de Felicidad adaptada de la prueba original, para ser administrada a los adultos mayores, que se ha denominado "Versión Adaptada de la Escala de Felicidad de Lima para adultos mayores", compuesta por 14 ítems o reactivos que permiten medir el constructo felicidad en sus cuatro dimensiones, con elevados índices de validez.

Para determinar el grado de confiabilidad, se trabajó con el coeficiente de confiablidad alfa de Cronbach, resultando que los valores para cada uno de los ítems de la escala son altamente significativos, con un rango de 0.82 y de 0.84 . Asimismo, la escala total presenta una alta consistencia interna $\alpha=0.84$. Estas puntuaciones muestran consistencia en la medición, es decir que existe una alta probabilidad de repetibilidad de las puntuaciones de la prueba en otras poblaciones de adultos mayores. 
TABLA 5

Estadísticos total-elemento

\begin{tabular}{lcccc}
\hline & $\begin{array}{c}\text { Media de la escala si se } \\
\text { elimina el elemento }\end{array}$ & $\begin{array}{c}\text { Varianza de la escala si } \\
\text { se elimina el elemento }\end{array}$ & $\begin{array}{c}\text { Correlación elemento- } \\
\text { total corregida }\end{array}$ & $\begin{array}{c}\text { alfa de Cronbach si se } \\
\text { elimina el elemento }\end{array}$ \\
\hline Ítem 1 & 51.57 & 74.172 & 0.336 & 0.839 \\
Ítem 2 & 51.52 & 73.497 & 0.323 & 0.842 \\
Ítem 3 & 51.84 & 69.475 & 0.532 & 0.827 \\
Ítem 5 & 51.32 & 73.426 & 0.452 & 0.832 \\
Ítem 6 & 51.29 & 69.661 & 0.64 & 0.821 \\
Ítem 8 & 51.42 & 71.472 & 0.486 & 0.83 \\
Ítem 9 & 51.84 & 69.324 & 0.481 & 0.831 \\
Ítem 10 & 51.3 & 68.4 & 0.624 & 0.821 \\
Ítem 12 & 50.8 & 74.65 & 0.536 & 0.83 \\
Ítem 16 & 51.05 & 71.441 & 0.651 & 0.823 \\
Ítem 17 & 51.56 & 71.192 & 0.446 & 0.833 \\
Ítem 18 & 51.46 & 72.251 & 0.424 & 0.834 \\
Ítem 21 & 51.27 & 71.369 & 0.574 & 0.825 \\
Ítem 26 & 51.37 & 72.633 & 0.36 & 0.839 \\
\hline
\end{tabular}

La escala total presenta una consistencia interna de $\alpha=0.84$.

Fuente: elaboración propia.

TABLA 6

Estadísticos de fiabilidad (Partición por Mitades)

\begin{tabular}{lllc}
\hline & Parte 1 & Valor & 0.724 \\
& & N de elementos & $7(\mathrm{a})$ \\
\cline { 2 - 3 } \multicolumn{1}{c}{ alfa de Cronbach } & Parte 2 & Valor & 0.684 \\
& & N de elementos & 7 (b) \\
\cline { 2 - 3 } Correlación entre formas & N total de elementos & 0.806 \\
\hline \multirow{2}{*}{ Coeficiente de Spearman-Brown } & & 0.892 \\
\hline Dos mitades de Guttman & Longitud igual & 0.892 \\
\hline
\end{tabular}

(a) Los elementos son los ítems: 1, 3, 5, 7, 9, 11 y 13.

(b) Los elementos son los ítems: 2, 4, 6, 8, 10, 12 y 14.

Fuente: elaboración propia.

También se determinó el coeficiente de partición por mitades de Spearman-Brown (longitudes iguales) y el de Guttman, resultando $r=0.89$ para el primero y $r=0.89$ para el segundo, lo que representa valores altos que demuestran una alta confiabilidad de la escala analizada.
El presente estudio sobre la validez y confiabilidad de la Escala de Felicidad de Lima constituye la fase inicial de una investigación que se propone medir la felicidad en adultos mayores venezolanos, para, en un futuro, buscar semejanzas y diferencias con la de los adultos mayores de otros países de Latinoamérica y del mundo. 


\section{Conclusiones}

Considerando que toda prueba o instrumento de carácter científico debe cumplir con el proceso técnico de validez, confiabilidad y pertinencia para su utilización en diferentes grupos, de acuerdo a las características de estos, se administró el instrumento original de Alarcón para medir felicidad a un grupo de 103 adultos mayores venezolanos, obteniéndose las propiedades psicométricas de dicho instrumento: validez y confiabilidad.

El alto índice de validez factorial resultante mediante el método de componentes principales para marcar el número de factores de la solución y la rotación Varimax, se reflejó en una alta saturación factorial en 14 items que fueron reagrupados en las cuatro dimensiones referidas por Alarcón. Este hallazgo muestra consistencia en la medición del constructo felicidad en adultos mayores, y apoya la evidencia del autor en cuanto a que la felicidad es un comportamiento multidimensional integrado por cuatro factores que se han denominado: Sentido positivo de la vida, Satisfacción con la vida, Realización personal y Alegría de vivir.

Los elevados índices de confiabilidad de alfa de Cronbach y de división por mitades de SpearmanBrown y Guttman alcanzados por el instrumento, evidencian una alta consistencia interna, es decir, precisión o repetibilidad de las puntuaciones. La eliminación de ítems de la escala originalmente construida y la reagrupación de los reactivos o ítems de mayor saturación, como resultado de la aplicación de la validez factorial, permitieron construir una escala para medir felicidad en adultos mayores que constituye una adaptación de la anterior. Se puede afirmar, por tanto, que la Escala Adaptada de Felicidad de Lima, compuesta por 14 ítems, presenta una alta confiabilidad de acuerdo a los coeficientes utilizados.

Los valores psicométricos obtenidos con la aplicación de la escala, la acreditan como un instrumento válido y confiable; así mismo, la muestra poblacional con la que se trabajó en su construcción permitió adaptarlo a las características de la población latina, por lo que se constituye en una herramienta que puede realizar importantes con- tribuciones al desarrollo de investigaciones sobre la felicidad en América Latina.

\section{Referencias}

Aiken, L. (2003). Tests psicológicos y evaluación (11. a ed.). México: Pearson Educación.

Alarcón, R. (2006). Desarrollo de una escala factorial para medir la felicidad. Revista Interamericana de Psicología, 40(1), 95-102.

Árraga, M. \& Sánchez, M. (2010). Bienestar subjetivo en adultos mayores venezolanos. Revista Interamericana de Psicología, 44(1), 1-8.

Chávez, N. (2001). Introducción a la investigación educativa. Maracaibo: Editorial Ars Gráficas S.A.

Cohen, J. \& Swerdlik, M. (2006). Pruebas y evaluación psicológica (6a. ed.). México: McGraw-Hill.

Cuadra, H. \& Florenzano, R. (2003). El bienestar subjetivo: hacia una psicología positiva. Revista de Psicología de la Universidad de Chile, 12(1), 83-96.

Diener, E., Suh, E. M., Lucas, R. E. \& Smith, H. L. (1999). Subjective well-being. Three decades of progress. Psychological Bulletin, 125(2), 276-302.

García, M. A. (2002, mayo). Desde el concepto de felicidad al abordaje de las variables implicadas en el bienestar subjetivo: un análisis conceptual. Revista Digital - Buenos Aires, 8(48), 4-6. Recuperado el 28 de febrero de 2008, de http://www.efdeportes. com/efd48/bienes3.htm

Gregory, R. (2001). Evaluación psicológica. Historia, principios y aplicaciones. México: Editorial El Manual Moderno.

Hernández, R., Fernández, C. \& Baptista, P. (2005). Fundamentos de la Metodología de la investigación (4a. ed.). México: McGraw-Hill Interamericana Editores.

Kerlinger, F. (2002). Investigación del comportamiento (4a. ed.). México: McGraw- Hill.

Neugarten, B. \& Tobin, S. (1999). Los significados de la edad. Barcelona: Herder.

Papalia, D. \& Olds, S. (2004). Psicología. México: McGraw-Hill Interamericana Editores.

Pelekais, C., Finol, M., Neuman, N. \& Parada, J. (2005). El ABC de la Investigación. Una aproximación teórico-práctica. Maracaibo: Ediciones Astro Data. 
Santrock, J. (2004). Introducción a la psicología. México: McGraw-Hill Interamericana Editores.

Yuste, N., Rubio, R. \& Aleixandre, M. (2004). Introducción a la psicogerontología. Madrid: Pirámide. 


\title{
Apéndice
}

\section{ESCALA DE FELICIDAD DE LIMA ADAPTADA A ADULTOS MAYORES}

\author{
(Alarcón, 2006; Árraga \& Sánchez, 2008)
}

\section{Datos Personales}

Iniciales de los nombres: Seudónimo:

Sexo: Hombre...... Mujer...... Edad...... Educación: Primaria ..... Secundaria ...... Superior...... Ocupación.

Estado civil: Soltero...... Casado...... Viudo...... Divorciado....... Conviviente...........

Ciudad donde vive:

Lugar donde vive (especificar si es su vivienda, de familiares, una institución u otros:

Personas con quienes vive:

Otra información relevante:

\section{Instrucciones}

Más abajo encontrará una serie de afirmaciones con las que usted puede estar de acuerdo, en desacuerdo o en duda. Lea detenidamente cada afirmación y luego utilice la escala para indicar su grado de aceptación o de rechazo a cada una de las frases. Marque su respuesta con X. No hay respuestas buenas ni malas.

Escala:

Totalmente de Acuerdo (TA)

Acuerdo (A)

$\mathrm{Ni}$ acuerdo ni desacuerdo (i)

Desacuerdo (D)

Totalmente en Desacuerdo (TD)

1. En la mayoría de las cosas mi vida está cerca de mi ideal.

TA A $i \quad$ D TD

2. Siento que mi vida está vacía.

TA A $i$ D TD

3. Las condiciones de mi vida son excelentes.

TA A $i$ D TD

4. La vida ha sido buena conmigo.

5. Me siento satisfecho con lo que soy.

TA A $i$ D TD

6. Hasta ahora, he conseguido las cosas que para mí son importantes.

TA A $i$ D TD

7. Si volviese a nacer no cambiaría casi nada en mi vida.

TA A $i$ D TD

8. Me siento satisfecho porque estoy donde tengo que estar.

TA A $\quad$ i $\quad$ D $\quad$ TD

9. Es maravilloso vivir.

TA A $i$ D TD

10. He experimentado la alegría de vivir.

TA A $i$ D TD

11. La vida ha sido injusta conmigo.

TA A $i \quad \mathrm{D}$ TD

12. Tengo problemas tan hondos que me quitan la tranquilidad.

TA A $i$ D TD

13. Estoy satisfecho con lo que hasta ahora he alcanzado.

TA A $i$ D TD

14. Todavía no he encontrado sentido a mi existencia.

TA A $i$ D TD

TA A $i$ D TD

\section{¡Gracias por su colaboración!}


